\title{
Wireless Networks and Internet of Things (IoT) Technologies based Localization System for Indoor Sports Player Performance Evaluation
}

\author{
J. A. Maldonado Cruz \\ Facultad de Ingeniería Eléctrica \\ Universidad Nacional Autónoma de México, 04510 \\ Ciudad de México, México
}

\author{
F. Moumtadi \\ Facultad de Ingeniería Eléctrica \\ Universidad Nacional Autónoma de México, 04510 \\ Ciudad de México, México
}

\begin{abstract}
This paper proposes the development of a player localization system using the Received Signal Strength Indicator (RSSI) parameter taken from IEEE $\mathbf{8 0 2 . 1 1}$ beacon frame, a positioning algorithm based on RSSI and trilateration technique to build a cost-effective solution for indoor sport environment as an alternative to video and Global Position System (GPS) localization technology. The visualization of the player's location on the court will be done through a web page.
\end{abstract}

Keywords - Indoor, Localization, RSSI, Trilateration.

\section{INTRODUCTION}

Players tracking helps coaches make informed decisions based on statistics to: preventing injuries, closely monitoring rehabilitation process, developing attacking and/or defending plays, as well as a better individual and collective performance of the players [1-4]. Though, there is the problem that in sports where players move quickly it is extremely difficult to analyze the sudden movements of the players without the help of technology. To carry out a localization and tracking monitoring, coaches use video-based or GPS-based technologies [5-6], however, video-based technologies are complex and expensive [2]. Although GPS is the established standard used to estimate the location of players in different sports for a quantitative test, it is not suitable for a precise local positioning, due to obstruction of the sign by roof and wall. Furthermore, in the GPS there is a generalized technology for global positioning with speed of measurement and precision (without additional corrections) limited [5,7].

For this reason, multiple techniques have been suggested to indirectly obtain the local position of static and / or moving objects with techniques such as: trilateration, multilateration, triangulation and proximity all of which are based on radiofrequency (RF) [8]. The main distance estimation parameters related to these techniques are: time of arrival (ToA), arrival time difference (TDoA), arrival angle (AoA) and Received Signal Strength Indicator (RSSI). The current location systems use these techniques individually or combined mainly in indoor environments such as houses and industries where there is a significant disorder of objects [9]. The advantage of bringing these systems to indoor sports is that, by not having objects interfering in the signals path except for the players themselves like in basketball [2], there is less effect from phenomes such as fading, scattering and reflections resulting in a better position estimate. Also, it is possible to use the IEEE 802.11 standard [10] standard because the 2.4 and $5 \mathrm{GHz}$ spectrum is not so saturated in training sport environments. RF localization techniques based on RSSI tend to be very accurate for short distances, but they are imprecise when increasing a few meters. However, as the RSSI parameter is very practical, it is still used by many researchers [7].

In this context, this paper presents the development of a location system for a transmitting beacon that will be carry by a player in indoor sport environment. A localization model will be made for the closed environment, the trilateration technique will be implemented, and the player localization with respect to the court will be shown in a two-dimensional representation by IoT technologies. The rest of the paper is organized as follows. In section II, a general description of the methodology will be presented. The results are shown in Section III. Finally, in section IV conclusions will be presented.

\section{STUDY DESIGN AND METHODOLOGY}

In order to develop an accurate positioning system is essential to carry out a study of RF signals propagation; nowadays there are currently many propagation models oriented to indoor environments [8,9] such as the RSSI parameter which is very practical [7] and useful since the unique obstacles in game fields are the players; therefore a model that employs this parameter was used. RSSI is defined as ten times the logarithm of the ratio power of the received signal and a reference power.

$$
\mathrm{RSSI}=10 \log (\mathrm{P} / \text { Pref })
$$

Furthermore, the power of the received signal is inversely related to the distance, i.e., $\operatorname{RSSI} \alpha\left(1 / \mathrm{D}^{\wedge} \mathrm{n}\right)$. Where $D$ is the distance between the transmitting and receiving antenna and $n$ is the path loss exponent factor. Equation (1) is related to (2):

$$
\mathrm{RSSI}=-10 \mathrm{n} \log (\mathrm{D})+\mathrm{C}
$$

From (2) [11], it's seen that RSSI has a linear relationship with the logarithm of distance, where $\mathrm{C}$ is a fixed constant. Equation (2) can also be represented as (3):

$$
\mathrm{RSSI}=-\operatorname{mlog}(\mathrm{D})+\mathrm{C}
$$

Where $\mathrm{m}$ is the slope of the linear equation between RSSI and the logarithm of the distance, the path loss exponent factor $\mathrm{n}$ can be expressed as:

$$
\mathrm{n}=\mathrm{m} / 10
$$

From (3), the relation of RSSI and the distance between antennas is obtained, this equation can also be expressed as (5) to estimate position. 


$$
\mathrm{D}=10^{\wedge}((\mathrm{RSSI}-\mathrm{C}) / \mathrm{m})
$$

To determine $\mathrm{m}$ and $\mathrm{C}$ of (3), known distances versus RSSI measurements graph must be obtained under conditions determined by the indoor environment and by the $\mathrm{WiFi}$ protocols. The conditions of the indoor sport environment have a close similarity to the dimensions of the court game where the tests were accomplished, which were $18 \mathrm{~m} \times 9 \mathrm{~m}$. To obtain the distance estimation model (5), three stages were developed: Requirements along with specifications for devices, radiation pattern of transmitters and receivers as well as experiment.

\section{A. Requirements along with specifications for devices}

The system to develop the model of distance estimation is conformed by two devices, firstly; the one that the player will carry as an access point (transmitting node) secondly; a device that will filter the RSSI value of the beacon frame from the AP (receiving node). After the study of requirements and specifications; the pycom WiPy 2.0 device with the state of the art Espressif ESP32 chipset with omnidirectional antenna was chosen as the transmitting node due to it is oriented to Wi-Fi beacons position applications also it has the characteristics of being cheap, non-invasive and a device that does not affect the performance of players. It is important to specify for which sport the propagation model was designed, in this case it will correspond to the training sessions of volleyball players in indoor game courts, which implies that the transmitting node can only be carried out in specific areas of the player's body; thus it is sure that performance will not be affected. Then; it was decided to place the transmitting node at the level of the player's back. For the reason that it is primarily designed for training sessions of 1 hour to 2 hours, the module WiPy 2.0 does not present problems when is powered with a lithium battery of $3.3 \mathrm{~V}-500 \mathrm{mAh}$; The module WiPy 2.0 has a low energy consumption of $0.5 \mathrm{~mW}$ configured as AP. For the receiver node; a module that runs Open Source OpenWrt system was selected with omnidirectional external dipole antennas; the receiver module was placed on a plastic base at a height of $0.3 \mathrm{~m}$ at ground level.

\section{B. Radiation pattern of transmitters and receivers}

To determine the orientation according to the receiving and transmitting node of the antennas, the radiation pattern of both antennas was determined in the game field, to determine in which direction, antennas had the highest RSSI sensitivity. The radiation pattern of the receiver node is shown in fig. 1 .

\section{Experiment}

The experiment was based on the displacement of the transmitting node with reference to the receiver node that remained fixed; the ideal condition in which the transmitting node moved without changing its original direction and the angle to obtain a distance estimation model according to (3). It is precisely to emphasize that the WiPy 2.0 module was configured with the $802.11 \mathrm{~b}$ standard. RSSI measurements were obtained against known fixed distances. In due course, with the maximum value of distance according to the position estimation obtained from the experiment, the optimal location of the three modules that act as receiver nodes were determined in the field to implement the trilateration technique.

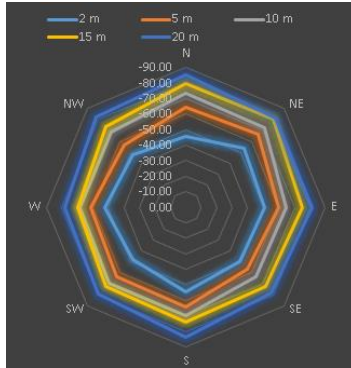

(a)

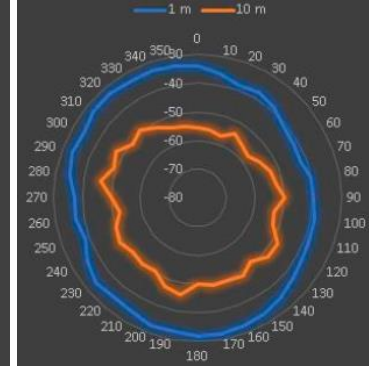

(b)
Fig. 1. (a) Radiation pattern of the receiver antenna, (b) Radiation pattern of the WiPy 2.0 antenna

\section{RESULTS}

As the RSSI values vary randomly due to time constraints, RSSI measurements were performed for 5 minutes every second, for 36 established distances (from $0.3 \mathrm{~m}$ to 18 $\mathrm{m}$, with intervals of $0.3 \mathrm{~m}$ and $0.5 \mathrm{~m}$ ). The RSSI values measured experimentally by the receptor node demonstrated a discrete behavior. From RSSI curves versus specific distances, the RSSI average result for each of the 36 curves was obtained, to later plot the experimental curve of RSSI vs average distance corresponding to fig. 2. The RSSI measurements revealed that after the theoretical distance of $10.5 \mathrm{~m}$ there is considerable uncertainty, so the maximum recommended distance estimation is $10.5 \mathrm{~m}$. If we consider that only the RSSI experimental values are up to $10.5 \mathrm{~m}$; a linear relationship was carried out among RSSI averaged measurement and the logarithm of the distance, so the coefficients $\mathrm{m}$ and $\mathrm{C}$ of (5) were obtained; The values of the coefficients are: $\mathrm{m}=-25.032$ and $\mathrm{C}=-39.632$.

$$
\mathrm{D}=10^{\wedge}((\mathrm{RSSI}+39.632) /-25.032)
$$

Furthermore, the path loss exponent factor for the indoor environment where the experiment was developed is equal to: $\mathrm{n}=-2.503$. Equation 6 represents the relationship between RSSI and distance for the indoor game court. With (6) and the RSSI average data collected from the experiment, the theoretical distances were calculated and compared with respect to the established distances in the experiment, the theoretical curve corresponding to (6) is shown in fig. 2. The error in the distance estimation between the AP and the receiver node can be obtained by comparing the theoretical and established distances in the experiment. Table 1 shows the error in the distance estimation when implementing (6). The error mean for the distance estimation model obtained in conditions where the WiPy 2.0 didn't change his direction and angle until $10.5 \mathrm{~m}$ is: $0.2444 \mathrm{~m}$.

To implement the trilateration technique, it's important to emphasize that, the antennas of the receiving nodes are the same and are dipole, therefore, under ideal conditions correspond to a circular pattern, assuming $\mathrm{z}=0$, the equation of each circle is:

$$
(\mathrm{x}-\mathrm{xi})^{\wedge} 2+(\mathrm{y}-\mathrm{yi})^{\wedge} 2=\mathrm{R}^{\wedge} 2
$$

Where (xi, yi) represents the location coordinate of each receiving node and $\mathrm{R}$ represents the radius, for a maximum estimation distance of 10.5 meters corresponding to the radius of the circular pattern of the antenna, an area of $9 \mathrm{~m} \times 9 \mathrm{~m}$ was established on the game court of $18 \mathrm{~m} \times 9 \mathrm{~m}$, in such a way that the receiver nodes are capable to fully cover an area to implement the trilateration technique as shown in fig. 3. 


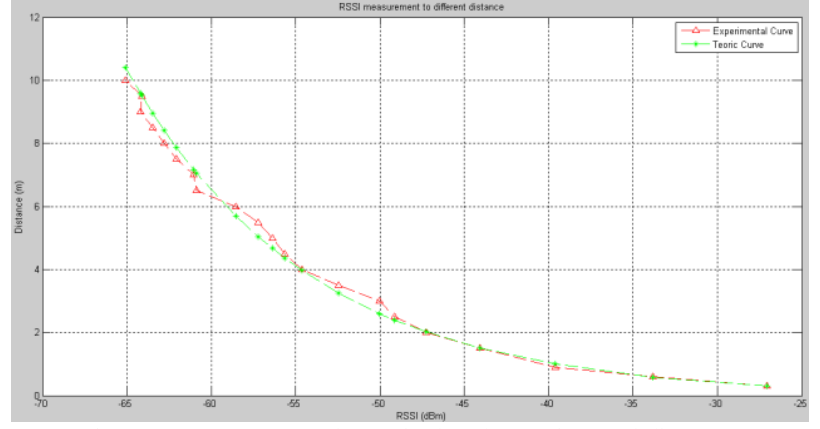

Fig. 2. RSSI curve vs. theoretical and experimental distance.

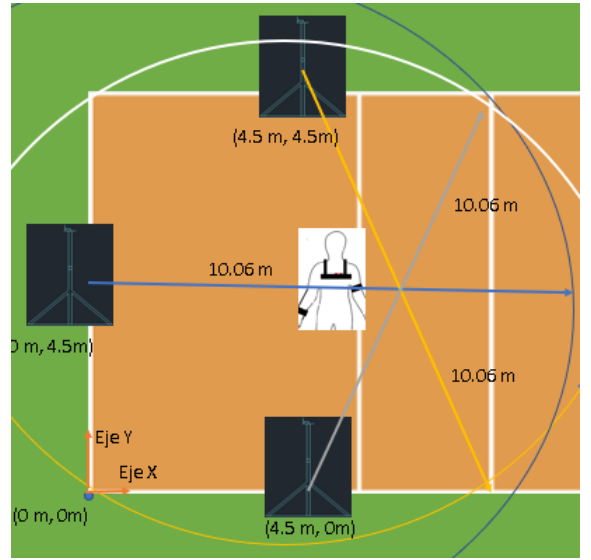

Fig 3. Implementation of the trilateration technique in the game court.

Equation (6) was implemented in each of the receiving nodes. Besides, the equations for trilateration according to fig. 3 are the following:

$$
\begin{aligned}
& (\mathrm{x})^{\wedge} 2+(\mathrm{y}-4.5)^{\wedge} 2=\mathrm{R} 1^{\wedge} 2 \\
& (\mathrm{x}-4.5)^{\wedge} 2+(\mathrm{y})^{\wedge} 2=\mathrm{R} 2^{\wedge} 2 \\
& (\mathrm{x}-4.5)^{\wedge} 2+(\mathrm{y}-4.5)^{\wedge} 2=\mathrm{R} 3^{\wedge} 2
\end{aligned}
$$

Based on the results, to fully cover the game court where the experiment has been carried out, the trilateration technique must be implemented 2 times, because the proposed trilateration technique can cover only an area of $9 \mathrm{~m} \times 9 \mathrm{~m}$.

Table 1. Error in the distance estimation

\begin{tabular}{|c|c|c|c|c|}
\hline \multirow{2}{*}{$\begin{array}{c}\text { Distance } \\
\text { (m) }\end{array}$} & \multicolumn{4}{|c|}{ Results } \\
\cline { 2 - 5 } & $\begin{array}{c}\text { Average } \\
\text { RSSI }(\mathrm{dBm})\end{array}$ & $\begin{array}{c}\text { RSSI Standard } \\
\text { deviation }(\mathrm{dBm})\end{array}$ & $\begin{array}{c}\text { Calculated } \\
\text { distance }(\mathrm{m})\end{array}$ & Error $(\mathrm{m})$ \\
\hline 0.3 & -27.0625 & 0.5040 & 0.3147 & 0.0147 \\
\hline 0.6 & -33.8437 & 1.2472 & 0.5872 & 0.0128 \\
\hline 0.9 & -39.625 & 1.0395 & 0.9994 & 0.0994 \\
\hline 1.5 & -44.0937 & 0.8175 & 1.5074 & 0.0074 \\
\hline 2 & -47.25 & 1.3137 & 2.0153 & 0.0153 \\
\hline 2.5 & -49.125 & 0.8032 & 2.3946 & 0.1054 \\
\hline 3 & -50.0312 & 1.0313 & 2.6028 & 0.3972 \\
\hline 3.5 & -52.4687 & 0.9832 & 3.2570 & 0.2430 \\
\hline 4 & -54.625 & 1.0395 & 3.9715 & 0.0285 \\
\hline 4.5 & -55.6562 & 1.4280 & 4.3667 & 0.1333 \\
\hline 5 & -56.375 & 1.2378 & 4.6651 & 0.3349 \\
\hline 5.5 & -57.2187 & 0.8322 & 5.0416 & 0.4584 \\
\hline 6 & -58.5312 & 0.9153 & 5.6886 & 0.3114 \\
\hline 6.5 & -60.875 & 1.0395 & 7.0572 & 0.5572 \\
\hline 7 & -61.0312 & 1.0621 & 7.1594 & 0.1594 \\
\hline 7.5 & -62.0625 & 0.8007 & 7.8718 & 0.3718 \\
\hline 8 & -62.78 & 1.6656 & 8.4088 & 0.4088 \\
\hline 8.5 & -63.4687 & 0.8418 & 8.9588 & 0.4588 \\
\hline 9 & -64.2135 & 0.6927 & 9.5941 & 0.5941 \\
\hline 9.5 & -64.125 & 1.0080 & 9.5163 & 0.0163 \\
\hline 10 & -65.0937 & 0.8175 & 10.4032 & 0.4032 \\
\hline & & & & \\
\hline
\end{tabular}

Equation (8), (9) and (10) were implemented in a RaspBerry $\mathrm{Pi} 3 \mathrm{~B}+$ that collected information from the receiving nodes, finally the position information of the player inside the court with coordinate format $(\mathrm{x}, \mathrm{y})$ was stored in a web server with tools of the IoT as seen in fig. 4.

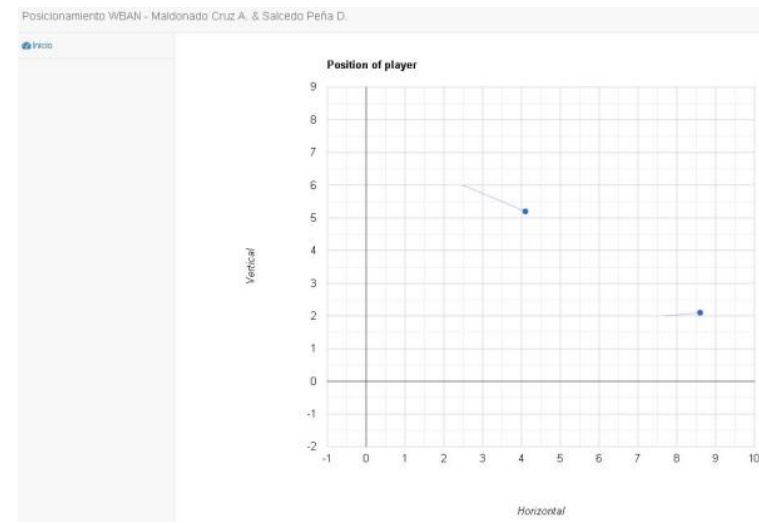

Fig. 4 Position display of two players in an area of $9 \mathrm{~m} \times 9$ on a web page

\section{CONCLUSIONS}

Therefore; the results show that AP position can be estimated with an average error of $0.24 \mathrm{~m}$ for a maximum distance of $10.5 \mathrm{~m}$ with RSSI monitoring the indoor sport environment where the experiment was achieved. The obtained error in the distance estimation measurement can be reduced by increasing height of antennas regarding the ground level, changing the $802.11 \mathrm{~b}$ standard of the WiPy 2.0 module to one with higher transmission power and consider the event in which the AP changes his direction and angle to gain a better accuracy of distance estimation by involving more parameters. Reducing the RSSI measurement uncertainty will be possible to get cover a greater area in the game court for distance estimation which requires fewer receiving nodes. The methodology to develop the proposed localization system is very useful because can be applied to any indoor sport.

\section{REFERENCES}

[1] E. Morais, S. Goldenstein, A. Ferreira and A. Rocha, "Automatic Tracking of Indoor Soccer Players Using Videos from Multiple Cameras," 2012 25th SIBGRAPI Conference on Graphics, Patterns and Images, Ouro Preto, 2012, pp. 174-181. DOI: 10.1109

[2] James A. Kirkup, "Real-time Wireless Player Positioning System in Basketball", COT, BEng, GradDip (Comp.), MIT, 2016.

[3] P. Rangsee, P. Suebsombat and P. Boonyanant, "Simplified low-cost GPS-based tracking system for soccer practice," 2013 13th International Symposium on Communications and Information Technologies (ISCIT), Surat Thani, 2013, pp. 724-728. DOI: 10.1109

[4] P. Wilhelm, E. Monier, P. Thomas y U. Ruckert, "SPA — A system for analysis of indoor team sports using video tracking and wireless sensor network," Proceedings of 6th International Symposium on Image and Signal Processing and Analysis, pp. 237-241, 2009.

[5] A. Stelzer, K. Pourvoyeur y A. Fischer, "Concept and application of LPM - a novel 3-D local position measurement system," IEEE Transactions on Microwave Theory and Techniques, pp. 2664-2669, 2004, DOI: 10.1109

[6] O. W. Ibraheem, A. Irwansyah, J. Hagemeyer, M. Porrmann y U. Rueckert, "Reconfigurable vision processing system for player tracking in indoor sports," Conference on Design and Architectures for Signal and Image Processing (DASIP), pp. 1-6, 2017, DOI: 10.1109.

[7] W. Chen, K. Kao, Y. Chang and C. Chang, "An RSSI-based distributed real-time indoor positioning framework," 2018 IEEE International Conference on Applied System Invention (ICASI), Chiba, 2018, pp 1288-1291. DOI: 10.1109 
[8] R. Xiong, S. van Waasen, C. Rheinlnder and N. Wehn, "Development of a Novel Indoor Positioning System With mm-Range Precision Based on RF Sensors Network," in IEEE Sensors Letters, vol. 1, no. 5, pp. 1-4, Oct. 2017, Art no. 5500504. DOI: 10.1109

[9] S. Liu, Y. Ma y J. Chai, "Research of three-dimensional indoor positioning based on RSSI," 9th International Congress on Image and Signal Processing (CISP-BMEI), pp. 1227-1231, 2016, DOI: 10.1109.

[10] M. S. Zehl, N. Karowski, A. Zubow and A. Wolisz, "LoWS: A complete Open Source solution for Wi-Fi beacon stuffing based Location-based Services," 2016 9th IFIP Wireless and Mobile Networking Conference (WMNC), Colmar, 2016, pp. 25-32. DOI: 10.1109

[11] T. A. Mounir, P. S. Mohamed, B. Cherif y B. Amar, "Positioning system for emergency situation based on RSSI measurements for WSN," International Conference on Performance Evaluation and Modeling in Wired and Wireless Networks (PEMWN), pp. 1-6, 2017. 